\title{
HIGHLIGHTS
}

(O) DEMENTIA

\section{Poor lung function in midlife predicts cognitive decline in men lacking APOE \&4}

New evidence suggests that impaired lung function in midlife is associated with cognitive decline in elderly men who lack the $\varepsilon 4$ allele of the apolipoprotein E gene ( $A P O E \varepsilon 4$ ), despite previous findings that individuals who carry this allele are at high risk of cognitive impairment and Alzheimer disease in later life. "A prior analysis showed that forced vital capacity predicted for completed suicide, [and] this led us to further analyze mental wellbeing and cognitive function in relation to lung function," explains lead author Erik Giltay.

The researchers, led by principal investigator Daan Kromhout, are involved in a long-term population-based cohort study as part of the Seven Countries Study, which was established to identify risk factors for heart attack and stroke. Forced vital capacity and forced expiratory flow was measured in 857 middle-aged men from Finland, Italy and the Netherlands in 1965. During 35 years of follow-up, the investigators have found a significant positive association between respiratory function and cognitive ability-Mini Mental State Examination (MMSE) scores recorded in 1990, 1995 and 2000-in individuals lacking $A P O E \varepsilon 4$ but not in carriers of this allele. This association remained significant following adjustment for body height.

Furthermore, although $A P O E \varepsilon 4$ carriership is known to increase the risk of dementia (a relationship confirmed in this study), the researchers found that forced vital capacity and forced expiratory flow correlated with cognitive function exclusively in APOE $\varepsilon 4$ noncarriers, according to the Clinical Dementia Rating criteria. Kromhout's team suggest that this relationship can be attributed to several factors, including reduced supply of oxygen to the brain or impaired fetal and postnatal development.

"As respiratory function can be objectively, reliably and easily assessed by spirometry, it may be utilized as a predictor of cognitive decline," concludes Giltay, who goes on to say, "further research is needed into the risk

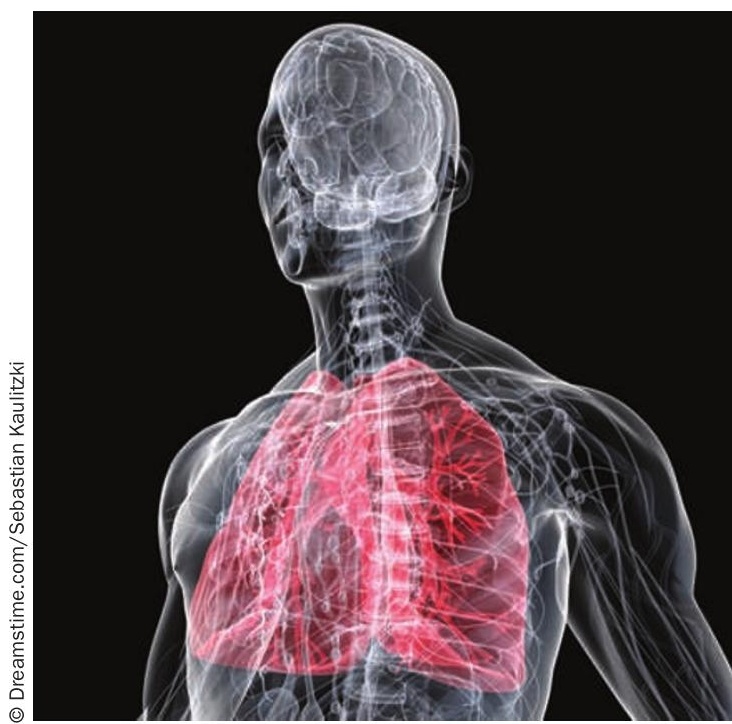

of dementia that may be modified by gene-environment interaction between the apolipoprotein E genotype and respiratory function."

Rowan Higgs

Original article Giltay, E. J. et al. Apolipoprotein E genotype modifies the association between midlife lung function and cognitive function in old age. Dement. Geriatr. Cogn. Disord. 28, 433-441 (2009) 